\title{
Viable inflationary models ending with a first-order phase transition
}

\author{
Marina Cortês ${ }^{1,2}$ and Andrew R. Liddle ${ }^{1}$ \\ ${ }^{1}$ Astronomy Centre, University of Sussex, Brighton BN1 9QH, United Kingdom \\ ${ }^{2}$ Berkeley Lab, Berkeley, CA 94720, USA
}

\begin{abstract}
We investigate the parameter space of two-field inflation models where inflation terminates via a first-order phase transition causing nucleation of bubbles. Such models experience a tension from the need to ensure nearly scale invariant density perturbations, while avoiding a near scale-invariant bubble size distribution which would conflict observations. We perform an exact analysis of the different regimes of the models, where the energy density of the inflaton field ranges from being negligible as compared to the vacuum energy to providing most of the energy for inflation. Despite recent microwave anisotropy results favouring a spectral index less than one, we find that there are still viable models that end with bubble production and can match all available observations. As a by-product of our analysis, we also provide an up-to-date assessment of the viable parameter space of Linde's original second-order hybrid model across its full parameter range.
\end{abstract}

PACS numbers: $98.80 . \mathrm{Cq}$

\section{INTRODUCTION}

One of the open questions in inflationary cosmology is the mechanism by which inflation came to an end. The current literature is dominated by two paradigms, violation of slow roll bringing inflation to an end while the field is still evolving, and a second-order phase transition of hybrid inflation type. However, Guth's original (but unsuccessful) proposal [1] invoked a first-order phase transition whereby inflation ended by nucleation of bubbles of true vacuum. First-order transitions have subsequently experienced bursts of popularity. In the late 1980s, La and Steinhardt 2] initiated intensive investigation of 'extended inflation' models, where modifications to Einstein gravity allowed bubble nucleation to complete in singlefield inflation. A few years later those models were struggling in face of observations, and focus instead returned to Einstein gravity, now in a two-field context with one rolling and one tunnelling field [3, 4, 5], although see [6, 7].

In addition to the usual quantum fluctuation mechanism, first-order inflation models produce density perturbations through the bubble collisions and subsequent thermalization. The spectrum of bubble sizes produced must be far from scale invariance to avoid clear violation with observed microwave anisotropies - the largest of the bubbles would otherwise be blatantly visible [8, 9, 10]. This requirement is typically at odds with the need to maintain scale invariance in the spectrum produced by quantum fluctuations, a tension sufficient to exclude extended inflation variants except in extremely contrived circumstances [11]. The purpose of this paper is to investigate whether the strengthened constraints of the post Wilkinson Microwave Anisotropy Probe (WMAP) era have eliminated the Einstein gravity first-order models too and, by implication, assess whether it is plausible that voids exist below current detection limits.

In Guth's original model, with one field, the inflaton must remain in the metastable vacuum long enough to allow for sufficient $e$-folds of inflation but in this case inflation never ends, the bubbles never thermalize and the transition doesn't complete. Introduction of a second field allows a time-dependent nucleation rate, permitting enough inflation to occur while the nucleation rate is low and a successful end when the rate rises to high enough values. This idea was proposed independently by Linde [3] and, in more detail, by Adams and Freese [4] under the name 'double-field inflation'.

Typically the second field, which is trapped in the metastable vacuum, also provides most of the energy density for inflation, although this depends on the particular values of parameters chosen. In that regime, the usual prediction is for a blue spectrum of density perturbations, $n_{\mathrm{S}}>1$. In the last few years the trend in cosmic microwave background (CMB) observations has been a tightening of the confidence limits around a central value $n_{\mathrm{S}}$ smaller than one, disfavouring this regime. Since our goal is to investigate the general viability of this type of model we will probe the entire parameter space, including the intermediate region where the contributions of each field to the energy density are comparable, making no approximations based on inflaton or the false vacuum domination.

As stated above one expects these models to run into difficulty with recent observations closing in on a nearly scale invariant scalar spectrum. CMB anisotropies observations place constraints on the maximum size of bubbles that survive from a first-order phase transition, at the time when scales of cosmological interest leave the horizon. In turn this places a strong upper limit on the nucleation rate at this time, after which it must rise sufficiently to complete the transition and provide a graceful exit for inflation. In order to meet these two requirements the field must proceed swiftly along the potential, what, in light of observations, places the model under stress. 


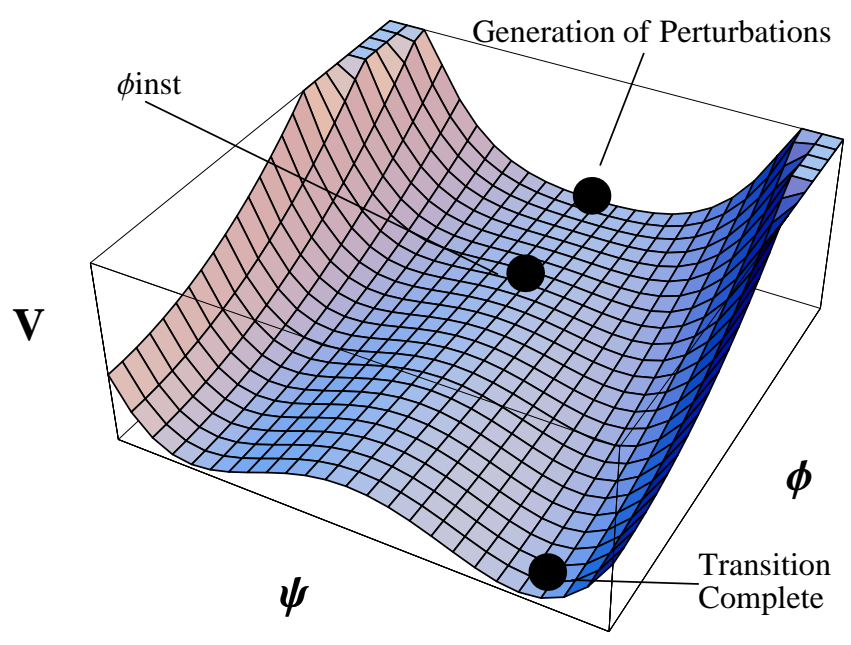

(a)

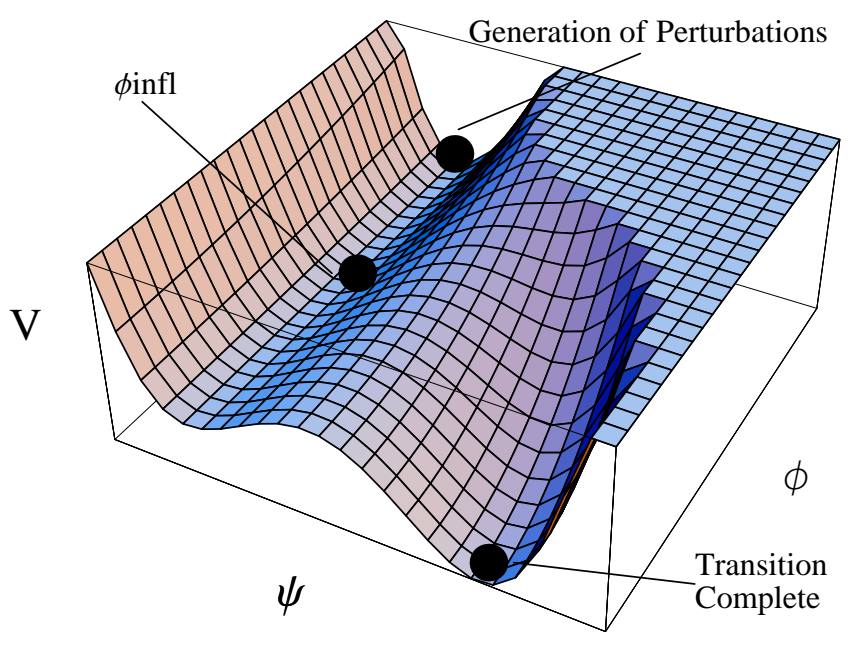

(b)

FIG. 1: (a) The potential for a second-order phase transition. The field reaches the true vacuum through a continuous transition, and the breaking of the symmetry implies that there will be defect formation at the end of the transition. The true vacuum minima develop once the field passes the point of instability, $\phi_{\text {inst }}$ (b) The same for the first-order case. In this case the transition is discontinuous and proceeds through quantum tunneling of the $\psi$ field to the true vacuum. The second minimum develops after the point of inflection $\phi_{\text {infl. }}$. The couplings in both (a) and (b) have been chosen so as to produce a visible barrier height (in working models this is negligible compared to the false vacuum energy).

\section{THE FIRST-ORDER MODEL}

We consider throughout a fairly general form of the potential for a first-order phase transition, given by Copeland et al [5].

$$
\begin{aligned}
V(\phi, \psi)= & \frac{1}{4} \lambda\left(M^{4}+\psi^{4}\right)+\frac{1}{2} \alpha M^{2} \psi^{2}-\frac{1}{3} \gamma M \psi^{3} \\
& +\frac{1}{2} m^{2} \phi^{2}+\frac{1}{2} \lambda^{\prime} \phi^{2} \psi^{2} .
\end{aligned}
$$

This extends the simplest second-order hybrid inflation model by addition of the cubic term for the $\psi$ field. As in conventional hybrid inflation, one envisages that initially the inflaton field $\phi$ is displaced far from its minimum, and the auxiliary field $\psi$ is then held in a false vacuum state by its coupling to the inflaton. Perturbations are generated during this initial phase as $\phi$ rolls slowly along the flat direction. The dynamics in this region are pretty much those of single-field slow-roll inflation, though the auxiliary field $\psi$ may provide most of the energy density for inflation, see Fig. [1]

In a model where the phase transition is second-order, shown in Fig. 1a, the false vacuum becomes unstable after $\phi$ passes a certain value, $\phi_{\text {inst }}$, and the fields evolve classically to their true vacuum (here producing topological defects as causally separated regions make independent choices as to which minimum to finish in). Although not the main topic of this paper, we explore current constraints on this model in the Appendix.

In the first-order case, shown in Fig. 10, if the parameters in Eq. (11) are chosen appropriately, a second minimum develops once the field evolves past a point of

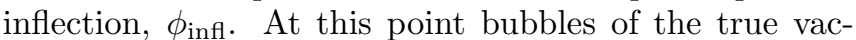
uum begin to nucleate and expand at the speed of light. The percolation rate is initially very small as the vacuum energies are comparable, but as $\phi$ approaches zero the interaction between the fields triggers a steep rise in the bubble production. Inflation ends when the nucleation rate reaches high enough values that the bubbles percolate and thermalize. In this case there is only one true vacuum and hence no topological defects. The channel in which the field rolls after tunnelling is much too steep to sustain any inflation within the bubbles.

For large values of $\phi$ there is only one minimum of the potential, and in the $\psi$ direction the potential looks like Fig. 22a. However if $\gamma^{2}>4 \alpha \lambda$, a second minimum develops after $\phi$ reaches a point of inflection

$$
\phi_{\text {infl }}^{2}=M^{2} \frac{\gamma^{2}-4 \alpha \lambda}{4 \lambda^{\prime}},
$$

as in Fig. 2 $\mathrm{b}$. The presence of the cubic term in the potential then breaks the degeneracy between the two minima, making it possible for the field to tunnel to the newly formed minimum. It is this second minimum that eventually becomes the true vacuum and the $\psi$ field begins to tunnel once the transition becomes energetically favourable, Fig. 2.

As mentioned in the previous section the quantum generation of perturbations occurs away from this minimum, while the inflaton is rolling in the $\phi$ direction, and we consider horizon exit to occur around $55 e$-folds before the end of inflation [12]. This evolution of $\phi$ is a cru- 


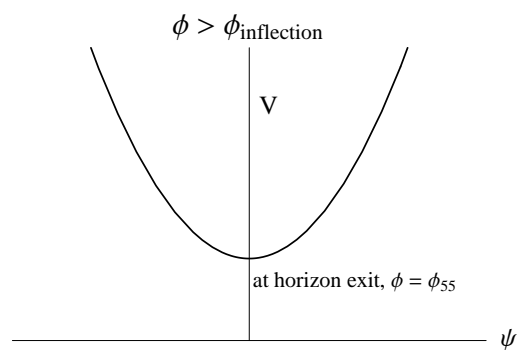

(a)

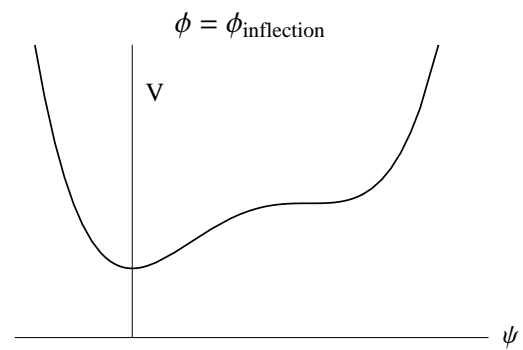

(b)

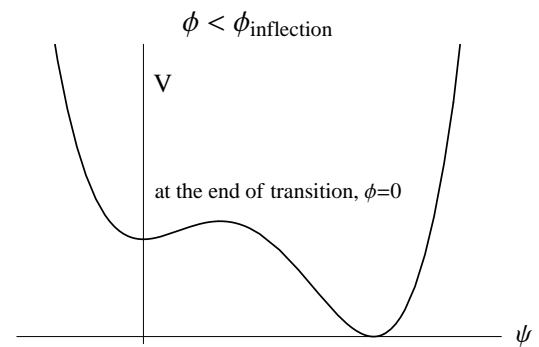

(c)

FIG. 2: (a) At early times, away from $\phi=0$, there is only minimum available for $\psi$ and the field is trapped in the false vacuum. (b) Given appropriate choices for the couplings a second minimum begins to develop when the field reaches the point of inflection of the potential. (c) Once the transition becomes energetically favourable the $\psi$ field begins to tunnel to the newly formed minimum, which eventually becomes the true vacuum.

cial feature of the model since it is the introduction of a time dependence in the tunneling rate that will allow the phase transition to complete, bringing inflation to an end.

The rate at which the bubbles nucleate is given by the percolation parameter (the number of bubbles generated per unit time per unit volume),

$$
p=\frac{\Gamma}{H^{4}} .
$$

In the limit of zero temperature (taken because the transition occurs during inflation) the nucleation rate of bubbles can be approximated by [13],

$$
p=\frac{\lambda M^{4}}{4 H^{4}} \exp \left(-S_{\mathrm{E}}\right),
$$

where $S_{\mathrm{E}}$ is the four-dimensional Euclidean action. $S_{\mathrm{E}}$ was obtained for first-order transition quartic potentials by Adams [14], who fitted the result as

$$
S_{\mathrm{E}}=\frac{4 \pi^{2}}{3 \lambda}(2-\delta)^{-3}\left(\alpha_{1} \delta+\alpha_{2} \delta^{2}+\alpha_{3} \delta^{3}\right),
$$

where $\alpha_{1}=13.832, \alpha_{2}=-10.819, \alpha_{3}=2.0765$, and $\delta$ is a monotonic increasing function of $\phi^{2}$,

$$
\delta=\frac{9 \lambda \alpha}{\gamma^{2}}+\frac{9 \lambda \lambda^{\prime} \phi^{2}}{\gamma^{2} M^{2}} .
$$

The allowed range has $0<\delta<2$ (outside this range solutions correspond to energetically disallowed transitions).

The transition to the true vacuum is complete once the percolation reaches unity, (one bubble per Hubble time per Hubble volume), allowing the bubbles of the true vacuum to coalesce.

However in the most general case inflation need not end through bubble nucleation. If the potential is too steep slow-roll is violated before bubbles thermalize and inflation ends before the transition completes. In this case the precise mechanism which completes the transition is irrelevant given that it occurs after inflation ends, and for our purposes the scenario is indistinguishable from the single-field case. (In this paper we do not consider gravitational waves produced via bubble collisions, but these may provide a further observable [15, 16, 17, 18] that can ultimately be used to constrain this type of model.)

The distinction between the two possibilities is given by the two values of the field, that at which the nucleation rate reaches unity, and that which makes $\epsilon \sim 1$ (violation of slow-roll), where $\epsilon$ is the usual slow roll parameter defined in Eq. (9). Inflation ends by whichever value of $\phi$ is reached first,

$$
\phi_{\text {end }}=\max \left(\phi_{\epsilon}, \phi_{\text {crit }}\right) .
$$

\section{INFLATIONARY DYNAMICS}

\section{A. Regimes}

Two different regimes can be distinguished, regarding which field we wish to have dominate the energy density. In the usual hybrid inflation regime the energy density of the potential is dominated by the false vacuum $\lambda M^{4} \gg m^{2} \phi^{2}$, which provides the energy for inflation. In the opposite regime, in which the inflaton dominates the energy density, the dynamics rapidly approach those of single-field inflation since, as we will see, slow-roll violation occurs sooner.

Working in either of these two regimes would allow us to simplify some of the expressions governing the dynamics during inflation, such as the number of $e$-folds and the slow-roll parameters, Eqs. (12), (9) and (15), and to proceed via an analytical treatment instead of a numerical one. However our purpose here is to probe the dynamics of the full $n_{\mathrm{S}}-r$ parameter space, $(r$ is the tensor-toscalar ratio given by Eq. (14)) so as to determine whether there still remain models consistent with CMB observations. Hence we also include the intermediate regime in our analysis, where the energy densities of the two fields are comparable, particularly when the transition between slow roll violation and bubble nucleation occurs. For this 
reason we will retain the full form of the potential and proceed through numerical calculations.

\section{B. Field dynamics}

In order to specify the dynamics of each model we begin by finding the field value, $\phi_{\max }$, at which inflation ends so we need to determine $\phi_{\epsilon}$ and $\phi_{\text {crit }}$. $\phi_{\epsilon}$ is obtained by evaluating the first slow-roll parameter for our potential and taking it to unity, ${ }^{1}$

$$
\epsilon \equiv \frac{m_{\mathrm{Pl}}^{2}}{16 \pi}\left(\frac{V^{\prime}}{V}\right)^{2}=\frac{m^{4} \phi^{2} m_{\mathrm{Pl}}^{2}}{\pi\left(\lambda M^{4}+2 m^{2} \phi^{2}\right)^{2}} \approx 1
$$

Inverting for $\phi$ yields,

$$
\phi_{\epsilon}^{2}=\frac{m^{2} m_{\mathrm{Pl}}^{2} \pm m m_{\mathrm{Pl}} \sqrt{m^{2} m_{\mathrm{Pl}}^{2}-8 \pi \lambda M^{4}}-4 \pi \lambda M^{4}}{8 \pi m^{2}}
$$

and we take the largest value of $\phi$. Note that the solution exists only for large values of $m$, where $m^{2} m_{\mathrm{Pl}}^{2}>8 \pi \lambda M^{4}$.

To determine $\phi_{\text {crit }}$ we need to find the value at which the percolation parameter reaches unity, $p_{\text {crit }} \sim 1$. Solving Eq. (4), we get

$$
S_{\text {crit }} \sim \ln \frac{\lambda M^{4}}{4 p_{\text {crit }} H^{4}}
$$

where $S_{\text {crit }}$ is given by Eq. (5).

Inverting Eq. (10) yields a value for $\phi_{\text {crit }}$ (only one of the three roots lies in the allowed range) and in turn this allows us to determine $\phi_{\text {end }}$, and, by comparison with $\phi_{\epsilon}$, the mechanism by which inflation ends.

Knowing $\phi_{\text {end }}$ we can calculate the value of the field at horizon exit, $\phi_{55}$. In this model $\phi$ rolls towards its minimum at $\phi=0$ so $\phi_{55}>\phi_{\text {end }}$. Using the expression for the number of $e$-folds between two field values $\phi_{1}$ and $\phi_{2}$ we get,

$$
N\left(\phi_{1}, \phi_{2}\right) \equiv \ln \frac{a_{2}}{a_{1}} \sim-\frac{8 \pi}{m_{\mathrm{Pl}}^{2}} \int_{\phi_{1}}^{\phi_{2}} \frac{V}{V^{\prime}} d \phi .
$$

For $\phi_{1}=\phi_{55}$ and $\phi_{2}=\phi_{\text {end }}$, and substituting for $V$, we have

$$
N\left(\phi_{55}, \phi_{\mathrm{end}}\right)=2 \pi \lambda \frac{M^{4}}{m^{2} m_{\mathrm{Pl}}^{2}} \ln \frac{\phi_{55}}{\phi_{\mathrm{end}}}+\frac{2 \pi}{m_{\mathrm{Pl}}^{2}}\left(\phi_{55}^{2}-\phi_{\mathrm{end}}^{2}\right),
$$

where we make no assumptions on the relative size of the two masses and retain both terms. Substitution of $\phi_{\text {end }}$ yields $\phi_{55}$ and now we can calculate the scalar spectral

\footnotetext{
1 The field $\psi$ sits in the false vacuum during the inflationary phase, since this is the only minimum available to $\psi$ in this region of the potential. This happens regardless of the means to ending inflation, so $\psi$ is set to zero throughout this section.
}

index, $n_{\mathrm{S}}$, and the tensor-to-scalar ratio, $r$, at horizon exit, by use of their expressions in terms of the usual slow-roll parameters,

$$
\begin{aligned}
n_{\mathrm{S}}-1 & =-6 \epsilon+2 \eta ; \\
r & =16 \epsilon,
\end{aligned}
$$

where $\epsilon$ is given by Eq. (9), and $\eta$ is

$$
\eta \equiv \frac{m_{\mathrm{Pl}}^{2}}{8 \pi} \frac{V^{\prime \prime}}{V}=\frac{m^{2} m_{\mathrm{Pl}}^{2}}{2 \pi\left(\lambda M^{4}+2 m^{2} \phi^{2}\right)},
$$

where the last equality is obtained by substitution of the potential.

At this point we can locate the model in the $n_{\mathrm{S}}-r$ plane and determine its position in relation to WMAP5 confidence limits [19].

\section{Choosing parameters}

Throughout we set the self-interaction and coupling constants, $\lambda$ and $\lambda^{\prime}$ respectively, equal to unity. We are then left with two constants, $\alpha$ and $\gamma$, and requiring the energy density of the true vacuum to be zero fixes one of these in terms of the other. We will fix $\alpha$ in terms of $\gamma$ but the reverse option could just as well be taken.

The CMB amplitude normalization can be used to relate the two masses. We use this to fix the mass of the light field $\phi$ and then we are left with only two undetermined parameters: the energy of the false vacuum, $M$, and the constant $\alpha$. For each value of $\alpha$, varying $M$ fully determines the dynamics of the fields, and describes a trajectory in the $n_{\mathrm{S}}-r$ plane shown in Fig. 3 .

Each line is composed of two branches which correspond to the two solutions of the WMAP normalization, and converge for large values of $M \sim 2.7 \times 10^{-3} m_{\mathrm{Pl}}$. For values of $M$ larger than this there is no solution to the amplitude normalization hence no viable models. This can be seen also in Fig. 4 which illustrates how the two different approximation schemes converge to a common behaviour and cease to exist after a certain value of $M$ (c.f. Fig. 1 of Ref. [5]).

The right-hand branch in Fig. 3 corresponds to the lower branch in Fig. 4 and to the smaller value of $m$ from the WMAP normalization. In this branch the approximate relation $M \sim m^{2 / 5}$ (in Planck units) holds and the false vacuum dominates. The dynamics are indistinguishable in the $n_{\mathrm{S}}-r$ plane when $M<10^{-4} m_{\mathrm{Pl}}$. We start with the typical slightly blue tilted spectrum and negligible tensor fraction. As $m$ continues to increase so does the deviation from $n_{\mathrm{S}} \sim 1$ until the approximate relation between the two masses breaks down and we have the inflaton playing a more significant role in the relative contribution of the two fields. At this point we observe a turn in the $n_{\mathrm{S}}-r$ plane, and the solution enters the intermediate region of comparable field energy densities.

Despite this we still observe inflation ending by bubble nucleation throughout this branch, from small values of $M$ to the maximum at $M \sim 2.7 \times 10^{-3} m_{\mathrm{Pl}}$. 


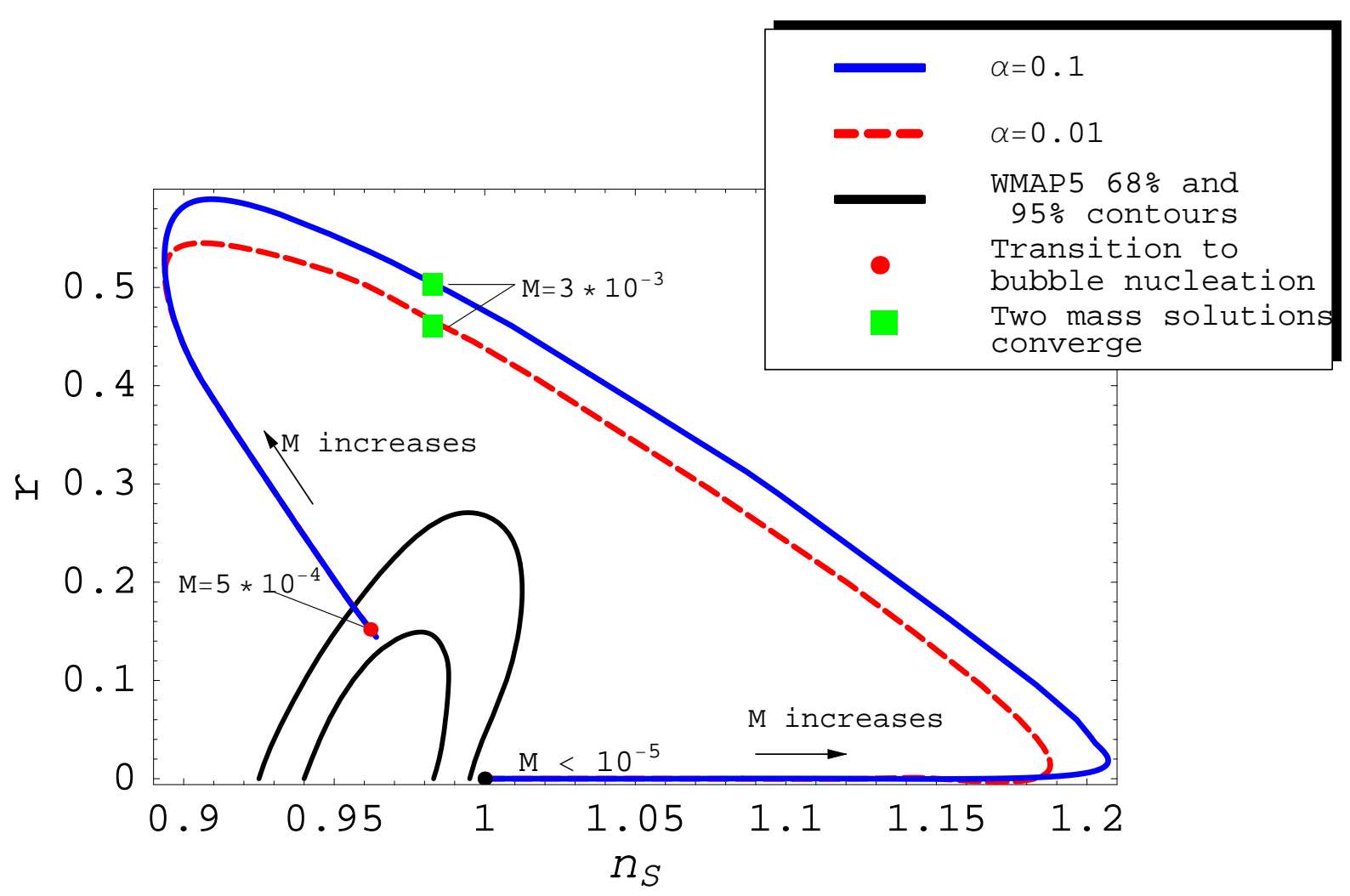

FIG. 3: The trajectories described in the $n_{\mathrm{S}}-r$ parameter plane for first-order models when $M$ is varying and $m$ is set by the CMB normalization. The two lines correspond to different values of the coupling constant $\alpha$, the outermost $\alpha=0.1$ and the innermost $\alpha=0.01$. The two endpoints correspond to the endpoints in Fig. 4 and converge at $M \sim 10^{-3} m_{\mathrm{Pl}}$ corresponding to the union of the branches in Fig. 4 at $\left(n_{\mathrm{S}}, r\right) \sim(0.99,0.5)$. Mass values are given in Planck units.

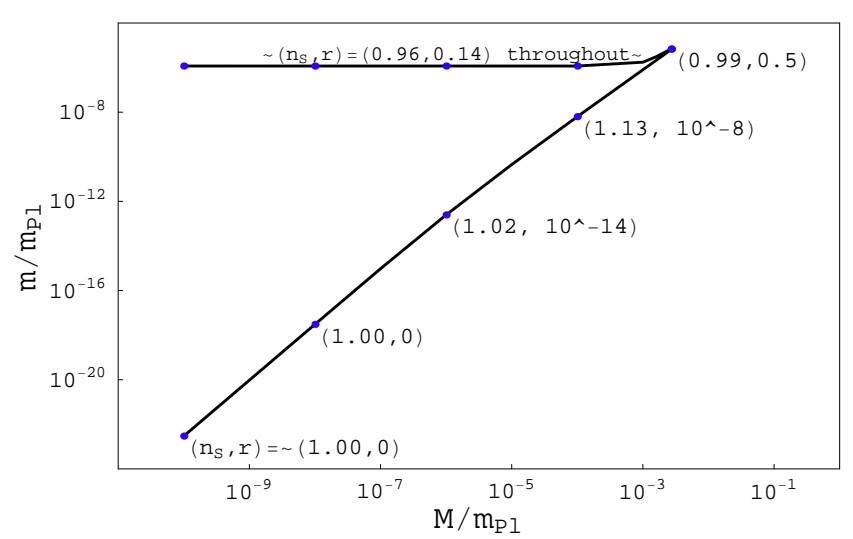

FIG. 4: The relation between the two mass scales. The WMAP normalization admits two solutions for $m$, corresponding to false vacuum domination over the inflaton (lower branch), and the opposite regime, for large $m$, which is nearly independent of $M$ (upper branch). The two regimes converge to common behaviour. We consider all three regimes in the analysis and set $\alpha=0.1$, given by the upper curve in Fig. 3

In the opposite branch, on the left-hand side, the model starts inside the WMAP5 95\% confidence contour, well inside the inflaton dominated regime. Similarly to the other branch we observe an initial period where there is little dependence on the false vacuum energy, corresponding to the plateau on Fig. 4, and the dynamics are very well approximated by those of standard single-field inflation with a $\phi^{2}$ potential, well known to satisfy WMAP5 data.

This regime breaks down as the false vacuum energy increases and eventually we recover the regime where the phase transition triggers the end of inflation before the violation of slow roll, meaning we are again in the bubble production scenario. The interesting results here draw from the fact that the transition occurs inside the WMAP5 95\% confidence contour, making these viable models even away from false vacuum domination. Fig. 5 is a zoom of this region showing the field mass, $M$, at which the transition to bubble nucleation occurs, $M \sim 5 \times 10^{-4} m_{\mathrm{Pl}}$, still allowed by the $95 \%$ confidence limits. 


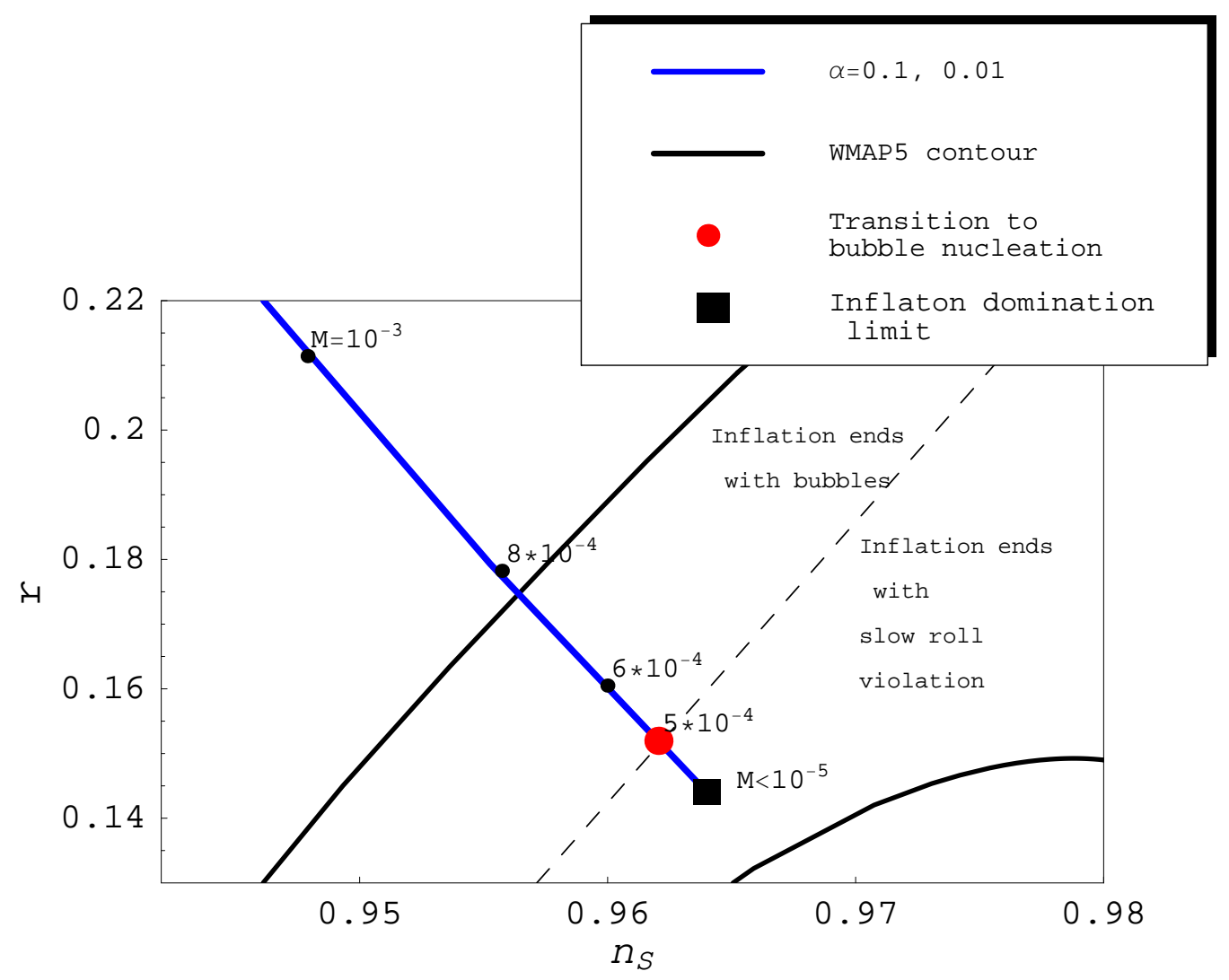

FIG. 5: Zoom of Fig. 3 showing the transition between models ending by slow roll violation and bubble inflation. The transition happens around $M \sim 5 \times 10^{-4} m_{\mathrm{Pl}}$, well within the WMAP5 95\% confidence contour. Mass values in Planck mass units.

\section{THREE CONSTRAINTS}

In the previous section we looked at constraints in the $n_{\mathrm{S}}-r$ plane. By specifying a value for $\alpha$, one of our two free parameters $(M, \alpha)$, the CMB normalization then allows us to recover a trajectory in this plane and assess where the density perturbations are compatible with WMAP5 data. We now compute other constraints on the scenario, in the $M-\alpha$ plane.

\section{A. Model consistency}

We begin with the requirement that $M$ be not larger than an upper limit above which, for a particular choice of couplings, the transition does not complete $\left(\phi_{\text {crit }}\right.$ does not exist). We call this the model consistency constraint, which translates to a relation for the value of $\phi_{\text {crit }}$, coming from the requirement that there exists a solution of Eq. (6) for $\delta$. Because of the constant term in Eq. (6) this is an additional requirement to $0<\delta<2$.

Since we have chosen to set $m$ by the CMB normalization this can be translated into an excluded region in the $(M, \alpha)$ plane (although alternatively we could have expressed it in terms of a region in $(M, m)$, by having $\alpha$ specified by the CMB normalization instead). This yields the region below the upper (blue) curve in Fig. 6. We see that specifying a value for the false vacuum density imposes an upper limit on the coupling $\alpha$ (alternatively on the inflaton mass, $m$ ) in order for the model to have the possibility to complete the phase transition.

\section{B. Big bubble constraint}

We adopt here a fairly crude criterion to judge whether the bubbles are compatible with observations, which is that any bubbles produced at the end of inflation and expanded to astrophysical sizes must, during the epoch of recombination, have a comoving size not larger than $20 h^{-1} \mathrm{Mpc}$ [8]. This corresponds to a maximum filling fraction at that time of $10^{-5}$, and puts an upper bound on the percolation rate of bubbles at the time the scales we observe today left the horizon:

$$
\left(\frac{\Gamma}{H^{4}}\right)_{55} \leq 10^{-5}
$$


With our form for the action Eq. (5) and choice of potential this becomes

$$
S_{55} \sim-2.9+4 \ln \frac{m_{\mathrm{Pl}}}{\lambda^{1 / 4} M}+11.5 .
$$

This gives us the region between the short dashed (black) lines in Fig. 6.

\section{WMAP constraint}

We can similarly place constraints on the $(M, \alpha)$ plane, by considering the $95 \%$ confidence limit resulting from the WMAP $5 n_{\mathrm{S}}-r$ plane when tensors are included.

$$
n_{\mathrm{S}} \lesssim 1.05
$$

Inverting Eq. (18) gives us an upper limit on $M$ in terms of $\alpha$, resulting in the region left of the long dashed (red) line in Fig. 6.

We also see from Fig. 6 that this constraint is opposed to that coming from the CMB maximum bubble size requirement, as we argued in Section I. Big bubbles at last scattering put an upper limit on the nucleation rate at horizon crossing while CMB constraints on the spectral tilt put a lower bound on the nucleation rate, from the requirement that $n_{\mathrm{S}}$ is not too distant from scale invariance.

Nevertheless, a region of parameter space survives all constraints.

\section{CONCLUSIONS}

Our principal conclusion is that there do remain Einstein gravity models of first-order inflation which are compatible with observations, despite the increasing tension between the need for a scale-invariant primordial spectrum and the suppression of large-scale bubbles. We have exhibited a particular class of model and found the parameter region where the first-order model is viable. Its predictions for $n_{\mathrm{S}}$ and $r$ are similar to the simple $m^{2} \phi^{2}$ slow-roll inflation model, though a little further from scale-invariance.

In this paper we have imposed a relatively simple constraint on the bubbles, and have then assumed that their impact on the CMB is negligible as far as constraints on the primordial perturbations are concerned. A more detailed treatment would combine the two perturbation sources and refit to the CMB data, which may lead to some modification to the outcome in regimes where the bubble production is close to the observational limit. For models where the bubbles are safely within the observational limits this is not an issue.

This paper demonstrates that we are still some way from having a clear view as to how the inflationary period of the Universe may have ended. The literature contains three different mechanisms - violation of slow-roll, a second-order instability during slow-roll, and bubble nucleation - and we have shown that the last (and least popular) of these remains a viable option. First-order models are of phenomenological interest as the bubble spectrum is an additional source of inhomogeneity that could be considered in matching high-precision observations. The bubble collisions may also generate detectable gravitational waves $[15,16,17,18]$. There is therefore an ongoing need to refine understanding of the nature of perturbations induced by a primordial bubble spectrum.

\section{Acknowledgments}

M.C. was supported by FCT (Portugal) and by the Director, Office of Science, Office of High Energy Physics, of the U.S. Department of Energy under Contract No. DE-AC02-05CH11231. A.R.L. was supported by STFC (UK). We thank Andy Albrecht, Katie Freese, Andrei Linde, and Eric Linder for discussions and comments.

\section{APPENDIX A: THE SECOND-ORDER MODEL}

Although not part of our main study, the full parameter range of the second-order hybrid inflation model [5, 20, 21] is easily studied using the machinery we have used for the first-order case. The second-order model also uses the energy density of an auxiliary field to raise the energy scale for inflation without endangering slow roll. The phase transition in this case is continuous, with the $\psi$ field rolling down to the true vacuum (see Fig. 1(a)). There are no bubbles now and hence no bubble constraint; we just have to consider whether the usual perturbations are compatible with WMAP5 data. Furthermore since now there is no cubic term to break the degeneracy between the two minima, there is the possibility of topological defect formation at the end of inflation, as different regions in space roll towards one or the other minimum. However we do not consider their possible impact here.

The dynamics are closely related to those in the firstorder case. The critical point where the phase transition completes is a point of instability $\phi_{\text {inst }}$, after which $\psi=0$ becomes unstable and starts to roll. The potential for this case is a particularization of the first-order potential Eq. (11) with $\lambda=-\alpha$ and $\gamma=0$, and becomes,

$$
V(\phi, \psi)=\frac{1}{4} \lambda\left(\psi^{2}-M^{2}\right)^{2}+\frac{1}{2} m^{2} \phi^{2}+\frac{1}{2} \lambda^{\prime} \phi^{2} \psi^{2} .
$$

Apart from the expression determining $\phi_{\text {inst }}$, we can retain most of the expressions from the first-order model and build a similar picture in the $n_{\mathrm{S}}-r$ plane. We present this in Fig. 7, again for $\lambda=\lambda^{\prime}=1$. We see that the false vacuum dominated regime, which has $n_{\mathrm{S}}>1$ and negligible $r$, lies entirely outside the WMAP5 allowed region, as does the main curve of the intermediate regime. Only once the trajectory heads towards the slow-roll limit 


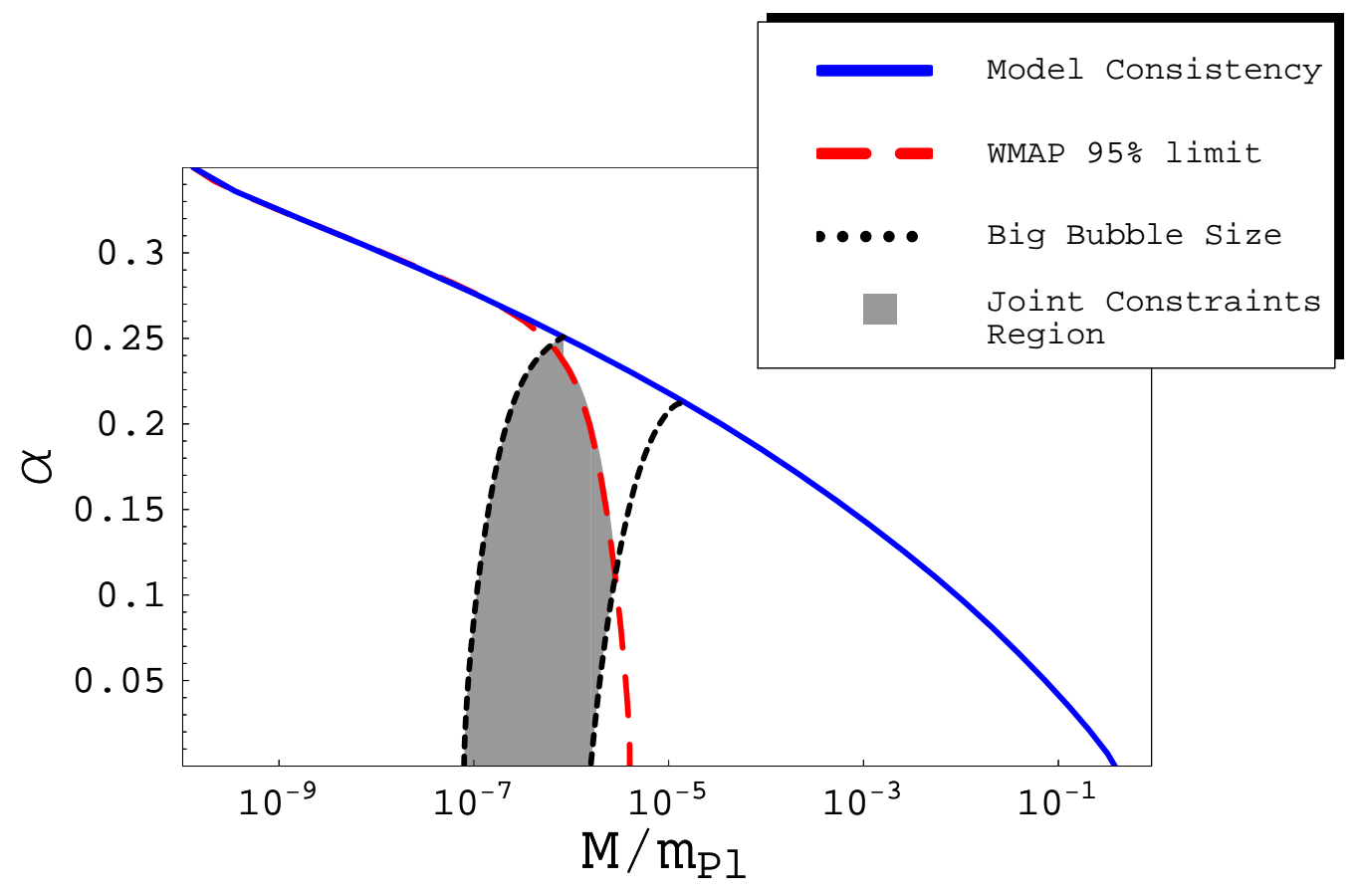

FIG. 6: Excluded regions in the $(M, \alpha)$ parameter space. The continuous (blue) line corresponds to ensuring model consistency; if the model lies above this region the phase transition will not take place. The long dashed (red) line corresponds to WMAP5 constraints on the value of the scalar perturbations tilt and allows models to the left of the bound. The region between the short dashed (black) lines indicates models satisfying the maximum size of bubbles allowed by the level of anisotropy in the CMB.

does it become compatible with observations. At $M \sim$ $9 \times 10^{-4} m_{\mathrm{Pl}}$ the models cross the WMAP5 $95 \%$ contour and at $M \sim 5 \times 10^{-4} m_{\mathrm{Pl}}$ inflation ends through slow roll violation instead of a phase transition.
[1] A. H. Guth, Phys. Rev. D23, 347 (1981).

[2] D. La and P. J. Steinhardt, Phys. Rev. Lett. 62, 376 (1989).

[3] A. D. Linde, Phys. Lett. B249, 18 (1990).

[4] F. C. Adams and K. Freese, Phys. Rev. D43, 353 (1991), hep-ph/0504135.

[5] E. J. Copeland, A. R. Liddle, D. H. Lyth, E. D. Stewart, and D. Wands, Phys. Rev. D49, 6410 (1994), astro$\mathrm{ph} / 9401011$.

[6] F. Di Marco and A. Notari, Physical Review D 73, 063514 (2006), astro-ph/0511396.

[7] T. Biswas and A. Notari, Physical Review D 74, 043508 (2006), hep-ph/0511207.

[8] A. R. Liddle and D. Wands, Mon. Not. Roy. Astron. Soc. 253, 637 (1991).

[9] A. R. Liddle and D. Wands, Phys. Lett. B276, 18 (1992).

[10] L. M. Griffiths, M. Kunz, and J. Silk, Mon. Not. Roy. Astron. Soc. 339, 680 (2003), astro-ph/0204100.

[11] A. R. Liddle and D. Wands, Phys. Rev. D45, 2665 (1992).
[12] A. R. Liddle and S. M. Leach, Phys. Rev. D68, 103503 (2003), astro-ph/0305263.

[13] C. G. Callan and S. R. Coleman, Phys. Rev. D16, 1762 (1977).

[14] F. C. Adams, Phys. Rev. D48, 2800 (1993), hepph/9302321.

[15] C. Hogan, Mon. Not. Roy. Astron. Soc. 218, 629 (1986).

[16] A. Kosowsky, M. S. Turner, and R. Watkins, Phys. Rev. D45, 4514 (1992).

[17] S. J. Huber and T. Konstandin, JCAP 0809, 022 (2008), arXiv:0806.1828.

[18] C. Caprini, R. Durrer, T. Konstandin, and G. Servant, Phys. Rev. D 79, 083519 (2009), arXiv:0901.1661.

[19] E. Komatsu et al. (WMAP), Astrophys. J. Suppl. 180, 330 (2009), arXiv:0803.0547.

[20] A. D. Linde, Phys. Lett. B259, 38 (1991).

[21] A. D. Linde, Phys. Rev. D49, 748 (1994), astro$\mathrm{ph} / 9307002$. 


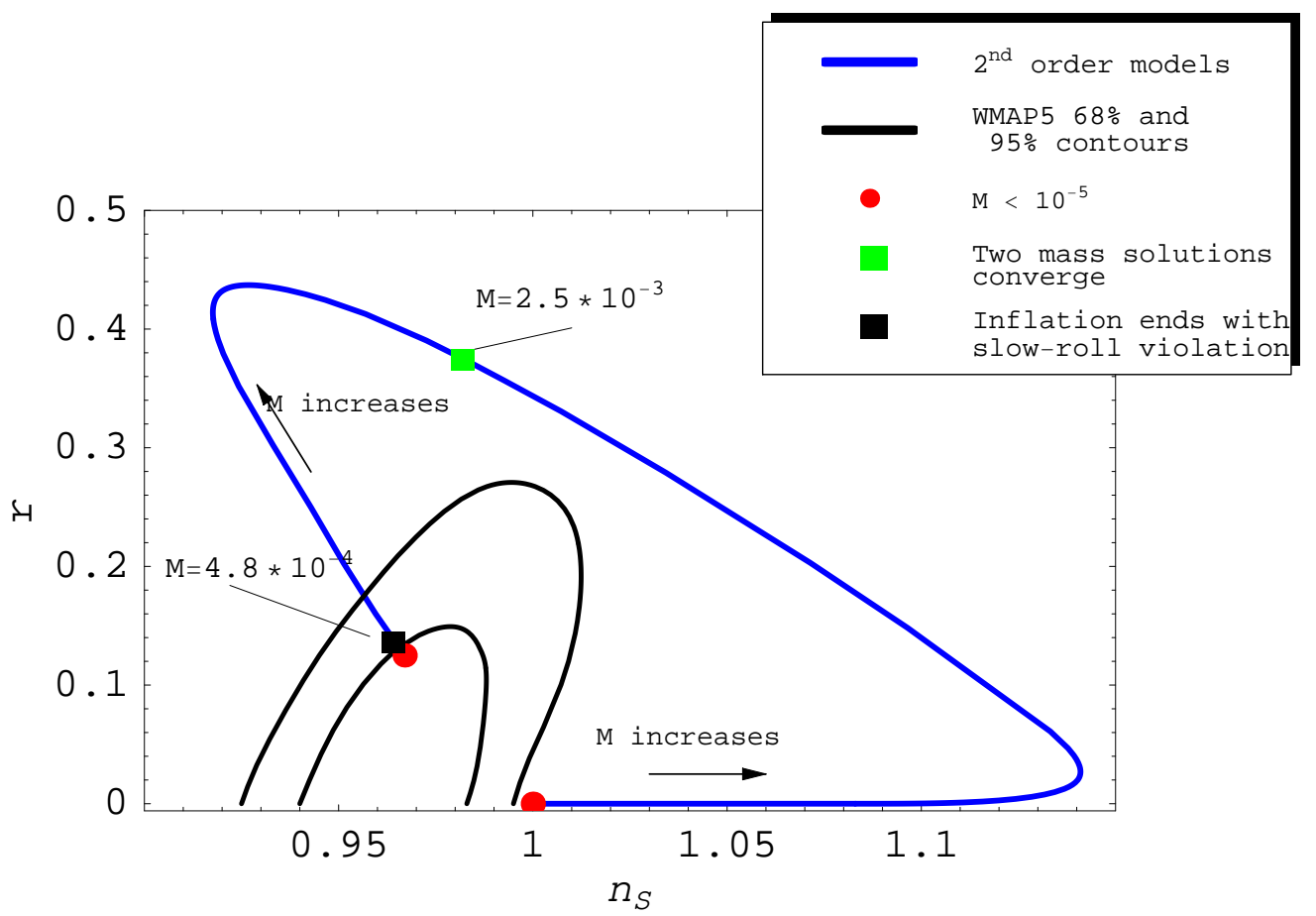

FIG. 7: Trajectory in parameter space $n_{\mathrm{S}}-r$ describing second-order hybrid inflation models when $M$ evolves from small through large values. The potential has one less coupling compared to the first-order case and all models are described by a single curve, as opposed to Fig. 3 Allowed models are those which approximate slow roll behaviour. False vacuum, blue tilted, models all lie outside the $95 \%$ C.L. 